\title{
Esophageal dislodgement of an endotracheal tube during nasopharyngeal oxygenation in a neonate with Pierre-Robin sequence: a case report
}

\author{
Mehmet K. Kanburoglu, M.D ${ }^{a}$, Mehmet N. Cizmeci, M.D ${ }^{a}$, Ahmet Z. Akelma, M.D ${ }^{a}$, Nebil Ark, M. $D^{b}$ and \\ Mustafa M. Tatli,M.D
}

\begin{abstract}
Conventional intubation for pulmonary management in children with a difficult airway may be very challenging even in skilled hands. Rapid advancements in respiratory care have reduced the incidence of difficult tracheal intubation and the incidence of complications have decreased accordingly. However, serious unexpected complications still occur in some patients today. Herein, we describe a syndromic newborn infant with a difficult airway who experienced migration of the endotracheal tube into the esophagus after displacement of the tube connector. We would like to share our experience and contribute to the literature with the presentation of this undesired event. To the best of our knowledge, no such complication has been reported in the neonatal literature before. Key words: Complication; nasopharyngeal intubation; neonate.
\end{abstract}

http:/ /dx.doi.org/10.5546/aap.2014.eng.e105

\section{INTRODUCTION}

Airway management as a part of pulmonary management is regarded as a safe procedure due to new techniques, technological developments and extensive research on this field. These rapid advancements in respiratory care have reduced the incidence of difficult tracheal intubation and the incidence of complications have decreased accordingly. ${ }^{1}$ However, serious unexpected complications still occur in some patients today. ${ }^{2}$ We have recently encountered a syndromic newborn infant with a difficult airway and experienced an unforeseeable complication of nasopharyngeal oxygenation. We would like to share our experience and contribute to the literature with the presentation of this undesired event.

a. Department of Pediatrics.

b. Department of Otorhinolaryngology.

Fatih University Medical School, Ankara/Turkey.

Correspondencia:

Mehmet Nevzat Cizmeci, M.D: nevzatcizmeci@gmail.com

Conflict of interest: None.

Recibido: 24-10-2013

Aceptado: 2-1-2014

\section{Case presentation}

A $2770 \mathrm{~g}$ male infant was born at 36 weeks' gestation; his mother was 31-year old, gravida 2 , para 1. Upon delivery, the infant showed marked cyanosis and difficult breathing. There was a significant retrognathism, cleft palate and glossoptosis; all signs were consistent with PierreRobin sequence. Since he was unresponsive to continuous positive airway pressure via nasal prongs, endotracheal intubation was planned by the neonatologist; however, repetitive attempts were unsuccessful due to obstruction of the airway with the downward displacement of the tongue and marked rigidity of the oral aperture.

Initial stabilization was performed with bag and mask ventilaton and oxygenation was maintained by nasal intermittent positive pressure ventilation after nasopharyngeal intubation. There was a good clinical response to this management and pulse-oximetry readings showed significant improvement. However, during follow-up the endotracheal tube was unanticipatedly dislodged into the esophagus after displacement of the tube connector while the infant was fighting the ventilator. Extensive efforts have failed to remove the tube by oral approach and the tube remained stuck between the esophagus and stomach (Figure 1). Endoscopical removal was executed under elective conditions by otorhinolaryngologists to prevent from a possible gastrointestinal obstruction and tracheostomy was performed for further management.

\section{DISCUSSION}

Conventional intubation in children with a difficult airway may be very challenging even in skilled hands and catastrophic complications including cardiac arrest, permanent brain damage and death following airway management is strikingly high. ${ }^{1,2}$ In the field of neonatology the principal conditions associated with a difficult airway are craniofacial syndromes related to maxillary and mandibular hypoplasia, and conditions which might cause limited mouth, jaw and neck mobility. ${ }^{3}$ However, with the 
introduction of major guidelines to overcome such a difficult airway scenario, the incidence of these complications have decreased substantially. ${ }^{2}$

Syndromic infants with facial dysmorphology as in this case might pose a challenge to the physicians during intubation. Additional experienced help needs to be called early after the first intubation attempt and it is important to consider whether any alternative methods or devices (i.e. fiberoptic tracheal intubation, laryngeal mask airways) are required. Supraglottic airways, especially laryngeal masks can effectively be utilized for airway maintenance for an extended period of time and for various medical and surgical procedures. Laryngeal masks also have an established role in the acute management of difficult airways for oxygenation and ventilation. ${ }^{3}$ Similarly, fiberoptic technique can be used safely in infants with a difficult airway. ${ }^{4}$ Nasopharyngeal placementof an endotracheal tube is a relatively simple approach, which can be used for oxygenation when intubation attempts have failed.5,6

Proper placement of the endotracheal tube during resuscitation can be challenging, especially in neonates and studies have shown the colorimetric end-tidal carbon dioxide detector to be helpful in confirming proper

FIgURE 1. Position of the dislodged tube between esophagus and stomach (arrows indicating the tips)

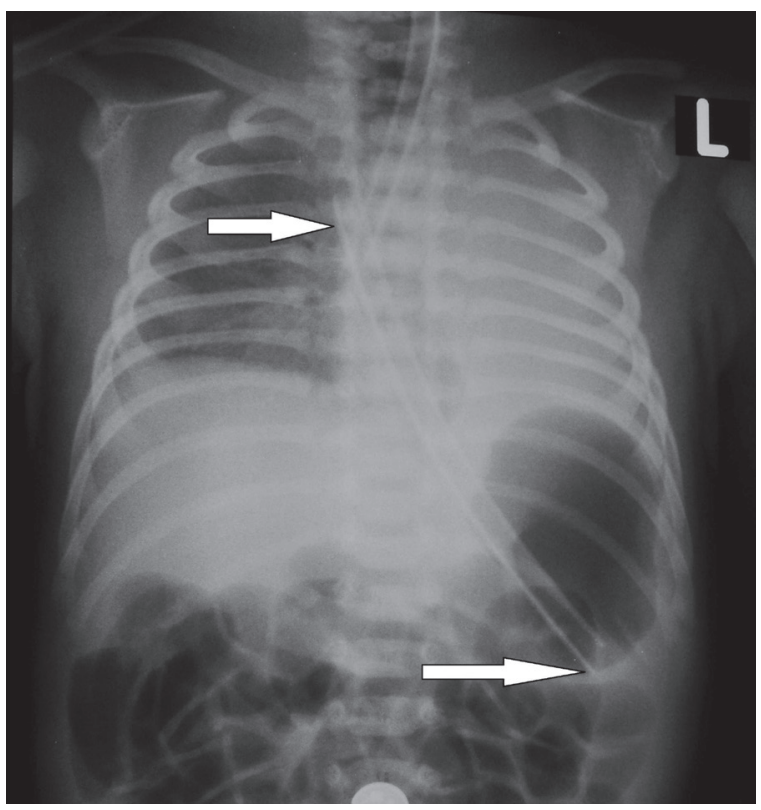

placement of the tube in hemodynamically stable patients. ${ }^{7}$ Hypoxia, aspiration, laryngospasm, laryngeal stenosis, tracheal rupture, cyst formation, pharyngoesophageal perforation are among the well-known complications of intubation in the neonatal period. ${ }^{8}$ However, exceptionally rare events regarding the use of endotracheal tubes might also occur. Endotracheal tubes are generally shortened to decrease the inner resistance occuring through the lumen. Plastic endotracheal tube connectors are then placed to the distal tip of the tube. These adapter parts might easily get separated from the tube if attached shallowly resulting in dislodgement. Moreover, the tube cannot be readily accessed for removal through the mouth in a syndromic infant due to the rigidity of the oral aperture.

We have presented this case to draw our colleagues' attention to such an undesired event. The whole course until removal of the tube was distressing for the attending physicians due to the skeptical attitudes of the parents towards medical malpractice. This type of unexpected displacement of the tube should be kept in mind and the connectors should be placed carefully when managing newborn infants. There are similar reports in the literature describing dislodgement of endotracheal tubes; 9,10 however, to the best of our knowledge, no such complication has been reported in the previous neonatal literature.

\section{Ethical Approval}

For this type of case report, no ethical permission is required in Turkey. Informed consent was taken from the parents for the publication of the case report.

\section{References}

1. Asai T. Strategies for difficult airway management--the current state is not ideal. J Anesth 2013;27(1):157-60.

2. Neyrinck A. Management of the anticipated and unanticipated difficult airway in anesthesia outside the operating room. Curr Opin in Anaesthesiol 2013;26(4):481-8.

3. Jagannathan E, Sequera-Ramos L, Sohn L, et al. Elective use of supraglottic airway devices for primary airway management in children with difficult airways. Br J Anaesth 2013;8 [in press].

4. Heard CM, Caldicott LD, Fletcher JE, Selsby DS. Fiberopticguided endotracheal intubation via the laryngeal mask airway in pediatric patients: a report of a series of cases. Anesth Analg 1996;82(6):1287-9.

5. Apfelbaum JL, Hagberg CA, Caplan RA, et al. Practice guidelines for management of the difficult airway: an updated report by the American Society of Anesthesiologists Task Force on Management of the Difficult Airway. Anesthesiology 2013;118(2):251-70. 
6. Weiss M, Engelhardt T. Proposal for the management of the unexpected difficult pediatric airway. Paediatr Anaesth 2010;20(5):454-64.

7. Gowda H. Question 2. Should carbon dioxide detectors be used to check correct placement of endotracheal tubes in preterm and term neonates? Arch Dis Child 2011;96(12):1201-3.

8. Cook TM, MacDougall-Davis SR. Complications and failure of airway management. Br J Anaesth 2012;109(Suppl 1):i68-i85.

9. Block EF, Cheatham ML, Parrish GA, Nelson LD, Beam N. Ingested endotracheal tube in an adult following intubation attempt for head injury. Am Surg 1999;65(12):1134-6.

10. GronczewskiCA. Thelostendotracheal tube: an unreported complication of prehospital intubation. Pediatr Emerg Care 2005;21(5):318-21. 